\title{
ARENS REGULARITY OF CERTAIN BANACH ALGEBRAS WHICH ARE IDEALS OF A BANACH ALGEBRA
}

\author{
SHEILA A. MCKILLIGAN
}

ABSTRACT. The question of Arens regularity for a dual $A^{*}$-algebra which is a dense two-sided ideal of a $B^{*}$-algebra has been discussed by Wong and by Alexander. We consider the Arens regularity of some Banach algebras which are dense ideals of Arens regular Banach algebras to prove, among other results, that any commutative $A^{*}$-algebra which is an ideal of its completion in the auxiliary norm is Arens regular.

1. Introduction. First of all we introduce our notation and then we establish some basic facts for later use. We let $\mathcal{U}$ be a Banach algebra throughout this section and we shall write $\mathfrak{U}^{*}$ and $\mathfrak{U}^{* *}$ for its first and second duals respectively.

For $\phi \in \mathfrak{U}^{* *}, F \in \mathfrak{U}^{*}$ and $x \in \mathfrak{U}$ we define $F_{x^{\prime}}, F^{x}, F_{\phi}, F^{\phi} \in \mathfrak{U}^{*}$ by

$$
\begin{aligned}
& F_{x}(y)=F(x y), \quad F^{x}(y)=F(y x) \quad(y \in \text { 歨 }), \\
& F_{\phi}(y)=\phi\left(F^{y}\right), \quad F^{\phi}(y)=\phi\left(F_{y}\right) \quad(y \in \mathfrak{U}) .
\end{aligned}
$$

For $\phi, \psi \in \mathfrak{U}^{* *}$ we define $\phi \cdot \psi, \phi \circ \psi \in \mathfrak{I}^{* *}$ by

$$
\phi \cdot \psi(F)=\psi\left(F_{\phi}\right), \quad \phi \circ \mho^{\prime}(F)=\phi\left(F^{\psi}\right) \quad\left(F \in \mathfrak{U}^{*}\right) .
$$

The products $\cdot$ and $\circ$ are the Arens products on $\mathfrak{U}^{* *}[2],[3],[5]$ and each of them makes $\mathfrak{O}^{* *}$ into a Banach algebra into which $\mathfrak{A}$ is embedded isometrically by the canonical homomorphism $a \mapsto \pi(a)(a \in \mathfrak{U}$ ) where $\pi(a)(F)=F(a)\left(F \in \mathfrak{U}^{*}\right)$.

We say that $F \in \mathfrak{U}^{*}$ is weakly almost periodic if $\phi \cdot \psi(F)=\phi \circ \psi(F)$ for all $\phi, \psi \in \mathfrak{U}^{* *}$. This is equivalent to the usual definition of weakly almost periodic [8]. The family of all weakly almost periodic functionals is denoted by $\mathfrak{C}(\mathfrak{U})$. Usually $\mathscr{C}(\mathfrak{U})$ is just a closed subspace of $\mathfrak{U}^{*}$. In the case $\mathfrak{I}(\mathfrak{X})=\mathfrak{U}^{*}$, we say that $\mathfrak{U}$ is Arens regular. Alternatively, $\mathscr{U}$ is Arens regular if $\phi \cdot \psi=\phi \circ \psi$ for all $\phi, \psi \in \mathfrak{U}^{* *}[5]$.

Received by the editors April 4, 1974.

AMS (MOS) subject classifications (1970). Primary 46H99.

Key words and phrases. Arens regular, $A^{*}$-algebra. 
Finally, the $w^{*}$ topology of $\mathfrak{U}^{*}$ is the topology induced in $\mathfrak{U}^{*}$ by $\mathfrak{U}$. We note that $\{\pi(a): a \in \mathfrak{U}\}$ is $w^{*}$ dense in $\mathfrak{U}^{* *}[7, \mathrm{p} .425]$.

We now set down some elementary facts which we shall use but for which we know no simple reference.

Proposition 1.1. Suppose $\left\{x_{\alpha}\right\},\left\{y_{\beta}\right\}$ are nets in $\mathfrak{U}$ with $\left\{\pi\left(x_{\alpha}\right)\right\},\left\{\pi\left(y_{\beta}\right)\right\}$ converging $w^{*}$ in $\mathfrak{U}^{* *}$ to $\phi, \psi$ respectively. For $F \in \mathfrak{U}^{*}$ we have

$$
\phi \cdot \psi(F)=\lim _{\beta} \lim _{\alpha} F\left(x_{\alpha} y_{\beta}\right), \quad \phi \circ \psi(F)=\lim _{a} \lim _{\beta} F\left(x_{\alpha} y_{\beta}\right) .
$$

Proof.

$$
\begin{aligned}
\phi \cdot \psi(F) & =\psi\left(F_{\phi}\right)=\lim _{\beta} \pi\left(y_{\beta}\right)\left(F_{\phi}\right)=\lim _{\beta} \phi\left(F^{y}\right) \\
& =\lim _{\beta} \lim _{a} \pi\left(x_{\alpha}\right)\left(F^{y}\right)=\lim _{\beta} \lim _{a} F\left(x_{\alpha} y_{\beta}\right) .
\end{aligned}
$$

A similar calculation establishes $\phi \circ \psi(F)=\lim _{\alpha} \lim _{\beta} F\left(x_{\alpha} y_{\beta}\right)$.

Corollary 1.2. If $F \in \mathbb{Q}(\mathscr{U})$, then

$$
\lim _{\beta} \lim _{\alpha} F\left(x_{\alpha} y_{\beta}\right)=\lim _{\alpha} \lim _{\beta} F\left(x_{\alpha} y_{\beta}\right)
$$

for every pair of nets $\left\{x_{\alpha}\right\},\left\{y_{\beta}\right\}$ in $\mathfrak{U}$ for which $\left\{\pi\left(x_{\alpha}\right)\right\},\left\{\pi\left(y_{\beta}\right)\right\}$ are $w^{*}$ convergent in $20 * *$.

Proof. Immediate from Proposition 1.1 and the definition of $\mathbb{Q}(2)$ ).

A net $\left\{e_{\gamma}\right\}$ in $\mathscr{U}$ is an approximate identity for $\mathscr{U}$ if $\lim _{\gamma}\left\|e_{y} x-x\right\|=0$ $=\lim _{\gamma}\left\|x e_{\gamma}-x\right\|(x \in \mathfrak{U})$ and $\left\|e_{\gamma}\right\| \leq M$ for all $\gamma$ where $M>0$.

Theorem 1.3. Suppose $\mathfrak{O}$ is Arens regular. If $\left\{e_{y}\right\}$ is an approximate identity for $\mathfrak{U}$, then there is a subnet $\left\{e_{\delta}\right\}$ with $\left\{\pi\left(e_{\delta}\right)\right\}$ converging $w^{*}$ in $\mathfrak{Q}^{* *}$ to $E$ which is the identity for $\mathfrak{U}^{* *}$ in the Arens multiplications.

Proof. It follows from the statement and proof of I.emma 3.8 of [5] that $\left\{e_{\gamma}\right\}$ has a subnet $\left\{e_{\delta}\right\}$ with $\left\{\pi\left(e_{\delta}\right)\right\}$ converging $w^{*}$ to $E$ which is a left identity for $\left(\mathfrak{U}^{* *},.\right)$ and a right identity for $\left(\mathfrak{U}^{* *}, 0\right)$. The result now follows from the Arens regularity and the uniqueness of a two-sided identity for $\mathfrak{Q}^{* *}$.

2. Banach algebras which are ideals in a Banach algebra. Let $(\mathfrak{U},||$ ) be a Banach algebra which is Arens regular and let $A \subset \mathscr{U}$ be a dense two-sided ideal of $\mathcal{U}$. Suppose further that $A$ is a Banach algebra 
under the norm \|\| and that, for $a \in A,|a| \leq C\|a\|$ where $C$ is a constant. Since $A \subset \mathscr{U}$ it will be convenient to denote the canonical embeddings of $A$, OU in $A^{* *}$, $\mathfrak{l}^{* *}$ respectively by $\pi_{A}, \pi$ respectively. This will be our standard notation throughout this section. We note that Barnes has studied such pairs of algebras (without the Arens regularity hypothesis) in [4] and we quote one of his theorems for ease of reference.

Theorem 2.1 [4, Theorem 2.3]. With A, Ul as above, there exists $D>0$ such that

(i) $\|a b\| \leq D\|a\||b|(a \in A, b \in \mathfrak{U})$, and

(ii) $\|a b\| \leq D|a|\|b\|(a \in \mathfrak{U}, b \in A)$.

Our aim is to show that, because $A$ is an ideal of $\mathfrak{A}$ and because of the relationship of the norms, translates of $F \in A^{*}$ can be extended to belong to $\mathfrak{U}^{*}$. The Arens regularity of $\mathscr{U}$ establishes the weak almost periodicity of such translates. In the case where the centre of $A$ contains an approximate identity of $(\mathfrak{Y},||)$ this is sufficient to give the weak almost periodicity of any $F \in A^{*}$ and so the Arens regularity of $A$. The result about commutative $A^{*}$-algebras follows.

Theorem 2.2. If $F \in A^{*}$, then, for $x \in A, \phi \in A^{* *}$, it is possible to extend $F_{x}, F^{x}, F_{\phi}, F^{\phi} \in A^{*}$ to belong to $\mathfrak{U}^{*}$.

Proof. Choose $x \in A$. Then, for $a \in A$,

$$
\left|F_{x}(a)\right|=|F(x a)| \leq\|F\|\|x a\| \leq D\|F\|\|x\||a| \quad \text { (Theorem 2.1). }
$$

Thus $F_{x}$ is bounded on $(A, \mid)$. Since $\mathscr{A}$ is the completion of $(A, \mid)$, we can extend $F_{x}$ to be bounded on $2 \mathbb{\text { with }}\left|F_{x}\right| \leq D\|F\|\|x\|$, where $\left|F_{x}\right|$ is the norm of $F_{x}$ as an element of $(\mathfrak{U},||)^{*}$. Similarly $F^{x}$ can be extended to belong to $(\mathfrak{U},||)^{*}$.

Choose $\phi \in A^{* *}$. Then, if $a \in A$,

$$
\left|F_{\phi}(a)\right|=\left|\phi\left(F^{a}\right)\right| \leq\|\phi\|\left\|F^{a}\right\| \text {. }
$$

But, for $b \in A$,

$$
\left|F^{a}(b)\right|=|F(b a)| \leq\|F\|\|b a\| \leq D\|F\|\|b\||a| \quad \text { (Theorem 2.1) }
$$

so that $\left\|F^{a}\right\| \leq D\|F\||a|$. Hence

$$
\left|F_{\phi}(a)\right| \leq D\|\phi\|\|F\||a|
$$

and $F_{\phi}$ is bounded on $(A,||)$. Thus $F_{\phi}$ may be extended to belong to $(\mathfrak{U},||)^{*}$ with $\left|F_{\phi}\right| \leq D\|\phi\|\|F\|$. Similarly $F^{\phi}$ can be extended to belong to $(\mathfrak{U},||)^{*}$. 
The next lemma provides the basic result on which much of our main result rests.

Lemma 2.3. Suppose $\left\{x_{\alpha}\right\},\left\{y_{\beta}\right\}$ are nets in $A$ with $\left\{\pi_{A}\left(x_{\alpha}\right)\right\},\left\{\pi_{A}\left(y_{\beta}\right)\right\}$ converging $w^{*}$ in $A^{* *}$ to $\phi, \psi \in A^{* *}$, respectively, and suppose that $\left\|x_{a}\right\| \leq\|\phi\|,\left\|y_{\beta}\right\| \leq\|\psi\|$. Then there exist subnets $\left\{x_{\gamma}\right\},\left\{y_{\delta}\right\}$ with $\left\{\pi_{A}\left(x_{\gamma}\right)\right\}$, $\left\{\pi_{A}\left(y_{\delta}\right)\right\}$ converging $w^{*}$ in $A^{* *}$ to $\phi, \psi$, respectively, and $\left.\left\{\pi_{\gamma}\right)\right\},\left\{\pi\left(y_{\delta}\right)\right\} w^{*}$ convergent in $\mathfrak{U}^{* *}$.

Proof. By our hypothesis about the norms || and \|\| , we have that

$$
\left|x_{\alpha}\right| \leq C\left\|x_{\alpha}\right\| \leq C\|\phi\| \text { and }\left|y_{\beta}\right| \leq C\left\|y_{\beta}\right\| \leq C\|\psi\|
$$

so that the nets $\left\{\pi\left(x_{\alpha}\right)\right\},\left\{\pi\left(y_{\beta}\right)\right\}$ are bounded in $\mathfrak{U}^{* *}$. It is immediate from Alaoglu's theorem [7, p. 424] that there are subnets $\left\{\pi\left(x_{\gamma}\right)\right\},\left\{\pi\left(y_{\delta}\right)\right\}$ converging $w^{*}$ in $2 \mathcal{L}^{* *}$. It is clear that $\left\{\pi_{A}\left(x_{\gamma}\right)\right\},\left\{\pi_{A}\left(y_{\delta}\right)\right\}$ are $w^{*}$ convergent in $A^{* *}$ to $\phi, \psi$ respectively.

We are now able to prove the interesting result that translates of $F \in A^{*}$ by elements of $A$ or of $A^{* *}$ are weakly almost periodic.

Theorem 2.4. If $\phi, \psi, \lambda \in A^{* *}, F \in A^{*}$ and $x \in A$, then

$$
\phi \cdot \psi\left(F^{x}\right)=\phi \circ \psi\left(F^{x}\right), \quad \phi \cdot \psi\left(F_{x}\right)=\phi \circ \psi\left(F_{x}\right),
$$

and

$$
\phi \cdot \psi\left(F^{\lambda}\right)=\phi \circ \psi\left(F^{\lambda}\right), \quad \phi \cdot \psi\left(F_{\lambda}\right)=\dot{\phi} \circ \psi\left(F_{\lambda}\right) .
$$

Proof. By Goldstine's theorem [7, p. 424] we can choose nets $\left\{x_{a}\right\}$, $\left\{y_{\beta}\right\}$ in $A$ with $\left\|x_{a}\right\| \leq\|\phi\|,\left\|y_{\beta}\right\| \leq\|\psi\|$ and such that $\left\{\pi_{A}\left(x_{\alpha}\right)\right\},\left\{\pi_{A}\left(y_{\beta}\right)\right\}$ converge $w^{*}$ in $A^{* *}$ to $\phi, \psi$ respectively. Using Lemma 2.3 , we choose subnets $\left\{x_{y}\right\},\left\{y_{\delta}\right\}$ with $\left\{\pi_{A}\left(x_{\gamma}\right)\right\},\left\{\pi_{A}\left(y_{\delta}\right)\right\}$ converging $w^{*}$ in $A^{* *}$ to $\phi, \psi$ and $\left\{\pi\left(x_{\gamma}\right)\right\},\left\{\pi\left(y_{\delta}\right)\right\}$ converging $w^{*}$ in $\mathfrak{Q}^{* *}$. Since $\mathscr{U}$ is Arens regular, it follows from Theorem 2.2 that $F^{x} \in \mathcal{W}(\mathfrak{U})$. By Corollary 1.2 it follows that

$$
\lim _{\delta} \lim _{\gamma} F^{x}\left(x_{\gamma} y_{\delta}\right)=\lim _{\gamma} \lim _{\delta} F^{x}\left(x_{\gamma} y_{\delta}\right)
$$

But, by Proposition 1.1 for $F^{x} \in A^{*}$, we have

$$
\phi \cdot \psi\left(F^{x}\right)=\lim _{\delta} \lim _{\gamma} F^{x}\left(x_{\gamma} y_{\delta}\right)
$$

and

$$
\phi \circ \psi\left(F^{x}\right)=\lim _{\gamma} \lim _{\delta} F^{x}\left(x_{\gamma} y_{\delta}\right)
$$


Hence

$$
\phi \cdot \eta\left(F^{x}\right)=\phi \circ \psi\left(F^{x}\right)
$$

The other equations are proved similarly.

Corollary 2.5. If $F \in A^{*}$, then $F_{x^{\prime}} F^{x}, F_{\phi}, F^{\phi} \in \mathcal{G}(A)$ whenever $x \in A$ and $\phi \in A^{* *}$.

We require one further lemma before our main result.

Lemma 2.6. If $(\mathfrak{U}, \mid)$ has an approximate identity $\left\{e_{\gamma}\right\}$ where each $e_{y}$ $\in A$, then, for $x, y \in A$,

$$
\lim _{\gamma}\left\|x y e_{\gamma}-x y\right\|=0=\lim _{\gamma}\left\|e_{\gamma} x y-x y\right\| .
$$

Proof. If $x, y \in A$, then

$$
\left\|x y e_{\gamma}-x y\right\|=\left\|x\left(y e_{\gamma}-y\right)\right\| \leq D\|x\|\left|y e_{\gamma}-y\right|
$$

by Theorem 2.1. But $\lim _{\gamma}\left|y e_{\gamma}-y\right|=0$, so $\lim _{\gamma}\left\|x y e_{\gamma}-x y\right\|=0$. Similarly $\lim _{\gamma} \| e y^{x y-x y \|}=0$.

Theorem 2.7. Suppose that $\mathcal{U}$ has an approximate identity $\left\{e_{y}\right\}$ where each $e_{\gamma} \in A$. Then there is a subnet $\left\{e_{\delta}\right\}$ of $\left\{e_{\gamma}\right\}$ such that, for $\phi, \psi \in$ $A^{* *}, F \in A^{*}$,

$$
\phi \cdot \psi(F)=\lim _{\delta} \phi \cdot \psi\left(F^{e}\right), \quad \phi \circ \psi(F)=\lim _{\delta} \phi \cdot \psi\left(F_{e_{\delta}}\right) .
$$

Proof. Choose nets $\left\{x_{a}\right\},\left\{y_{\beta}\right\}$ in $A$ such that $\left\|x_{a}\right\| \leq\|\phi\|,\left\|y_{\beta}\right\| \leq$ $\|\psi\|, \phi=w^{*} \lim _{a} \pi_{A}\left(x_{\alpha}\right), \psi=w^{*} \lim _{\beta} \pi_{A}\left(y_{\beta}\right)$ and $\left\{\pi\left(x_{\alpha}\right)\right\},\left\{\pi\left(y_{\beta}\right)\right\}$ converge $w^{*}$ in $\mathfrak{Q}^{* *}$ (possible by Lemma 2.3 and Goldstine's theorem [7, p. 424]). Let $\lambda, \mu \in \mathfrak{U}^{* *}$ with $\lambda=w^{*} \lim _{a} \pi\left(x_{\alpha}\right), \mu=w^{*} \lim _{\beta} \pi\left(y_{\beta}\right)$. Furthermore, by Theorem 1.3 we choose a subnet $\left\{e_{\delta}\right\}$ of $\left\{e_{\gamma}\right\}$ such that $\left\{\pi\left(e_{\delta}\right)\right\}$ converges $w^{*}$ in $\mathfrak{U}^{* *}$ to $E$, the identity of $\mathfrak{U}^{* *}$. We remark that we have, by Lemma 2.6,

$$
\lim _{\delta}\left\|x y e_{\delta}-x y\right\|=0=\lim _{\delta}\left\|e_{\delta} x y-x y\right\|
$$

whenever $x, y \in A$.

Now, by Proposition 1.1,

$$
\begin{aligned}
\phi \cdot \psi(F) & =\lim _{\beta} \lim _{a} F\left(x_{\alpha} y_{\beta}\right) \\
& =\lim _{\beta} \lim _{\alpha} \lim _{\delta} F\left(e_{\delta} x_{\alpha} y_{\beta}\right) \quad\left(\text { by }(*) \text { since } F \in A^{*}\right) \\
& =\lim _{\beta} \lim _{\alpha} \lim _{\delta} F^{y_{\beta}}\left(e_{\delta} x_{\alpha}\right)=\lim _{\beta} \lim _{\delta} \lim _{a} F^{y}\left(e_{\delta} x_{a}\right)
\end{aligned}
$$


by Corollary 1.2 since each $F^{y} \beta \in \mathcal{U}(\mathcal{U})$ (Theorem 2.2) and $\left\{\pi\left(e_{\delta}\right)\right\}$, $\left\{\pi\left(x_{\alpha}\right)\right\}$ are $w^{*}$ convergent in $\mathfrak{Q}^{* *}$.

But, by Proposition 1.1 and Theorem 2.2, for each $\beta$,

$\lim _{\delta} \lim _{\alpha} F^{y} \beta\left(e_{\delta} x_{\alpha}\right)=E \circ \lambda\left(F^{y} \beta\right)=\lambda \cdot E\left(F^{y} \beta\right) \quad$ (by Theorem 1.3)

$$
=\lim _{\delta} \lim _{\alpha} F^{y} \beta\left(x_{a} e_{\delta}\right)
$$

by Proposition 1.1. Hence

$$
\begin{aligned}
\phi \cdot \psi(F) & =\lim _{\beta} \lim _{\delta} \lim _{a} F^{y} \beta\left(x_{\alpha} e_{\delta}\right) \\
& =\lim _{\beta} \lim _{\delta} \lim _{\alpha} F^{e} \delta^{y} \beta\left(x_{\alpha}\right)=\lim _{\beta} \lim _{\delta} \phi\left(F^{e} \delta^{y} \beta\right) \\
& =\lim _{\beta} \lim _{\delta} F_{\phi}\left(e_{\delta} y_{\beta}\right)=E \cdot \mu\left(F_{\phi}\right)
\end{aligned}
$$

by Proposition 1.1 and Theorem 2.2. Now, by Theorem 1.3,E $\cdot \mu\left(F_{\phi}\right)=$ $\mu \cdot E\left(F_{\phi}\right)$ and, by Proposition 1.1,

Thus

$$
\mu \cdot E\left(F_{\phi}\right)=\lim _{\delta} \lim _{\beta} F_{\phi}\left(y_{\beta} e_{\delta}\right) .
$$

$$
\begin{aligned}
\phi \cdot \psi(F) & =\lim _{\delta} \lim _{\beta}\left(\pi_{A}\left(y_{\beta}\right) \cdot \pi_{A}\left(e_{\delta}\right)\right)\left(F_{\phi}\right) \\
& =\lim _{\delta} \lim _{\beta}\left(\left(\phi \cdot \pi_{A}\left(y_{\beta}\right)\right) \cdot \pi_{A}\left(e_{\delta}\right)\right)(F)=\lim _{\delta} \lim _{\beta}\left(\phi \cdot \pi_{A}\left(y_{\beta}\right)\right)\left(F^{e} \delta\right) \\
& =\lim _{\delta} \lim _{\beta} \pi_{A}\left(y_{\beta}\right)\left(\left(F^{e} \delta\right)_{\phi}\right)=\lim _{\delta} \phi \cdot \psi\left(F^{e} \delta\right) .
\end{aligned}
$$

A very similar calculation shows that $\phi \circ \psi(F)=\lim _{\delta} \phi \circ \psi\left(F_{e_{\delta}}\right)$. We then use Theorem 2.4 to establish $\phi \circ \psi(F)=\lim _{\delta} \phi \cdot \psi\left(F_{e_{\delta}}\right)$.

Immediate corollaries from this theorem are the following results which we state in full.

Corollary 2.8. Suppose ( $\mathscr{U},||)$ is an Arens regular Banach algebra and $A \subset \mathfrak{U}$ is a dense two-sided ideal of $\mathfrak{U}$. Suppose further that $(A,\|\|)$ is a Banach algebra with $|a| \leq C\|a\|(a \in A)$ for some constant $C$. If the centre of $A$ contains an approximate identity for $(\mathfrak{U}, \mid)$, then $(A,\|\|)$ is Arens regular.

Proof. In this case $F_{e_{\gamma}}=F^{e} \gamma$ whenever $F \in A^{*}$ and $\left\{e_{\gamma}\right\}$ is the approximate identity. 
Corollary 2.9. Suppose ( $\mathfrak{X},||)$ is a commutative Arens regular Banach algebra and $A \subset \mathfrak{U}$ is a dense ideal of $\mathscr{U}$. Suppose $(A,\|\|)$ is a Banach algebra with $|a| \leq C\|a\|(a \in A)$ for some constant $C$. If $A$ contains an approximate identity for $(\mathfrak{U},||)$, then $(A,\|\|)$ is Arens regular.

An $A^{*}$-algebra $(A,\|\|)$ is a Banach *-algebra which has an auxiliary norm || satisfying the $B^{*}$-algebra condition [9, p. 181]. The completion U of $A$ in || is therefore a $B^{*}$-algebra and we have the relation $|a| \leq C\|a\|$ $(a \in A)$ for some $C>0$ [9, p. 187]. The following theorem extends results about $A^{*}$-algebras proved by Wong [10] and Alexander [1].

Theorem 2.10. Suppose $A$ is a commutative $A^{*}$-algebra which is a dense ideal in its completion $\mathfrak{A}$ in the auxiliary norm. Then $A$ is Arens regular.

Proof. It follows from [6, Proposition 1.7.2] that $A$ contains an approximate identity of $\mathcal{U}$. Since any $B^{*}$-algebra is Arens regular [5, p. 869], the result is immediate from Corollary 2.9.

\section{REFERENCES}

1. F. E. Alexander, The dual and bidual of certain $A^{*}$-algebras, Proc. Amer. Math. Soc. 38 (1973), 571-576. MR 47 \#7459.

2. R. Arens, Operations induced in function classes, Monatsh. Math. 55 (1951), 1-19. MR 13, 372 .

3. - The adjoint of a bilinear operation, Proc. Amer. Math. Soc. 2 (1951), 839-848. MR 13, 659.

4. B. A. Barnes, Banach algebras which are ideals in a Banach algebra, Pacific J. Math. 38 (1971), 1-7. MR 46 \#9738; correction, ibid. 39 ( 1971 ), 828.

5. P. Civin and B. Yood, $T$ he second coniugate space of a Banach algebra as an algebra, Pacific J. Math. 11 (1961), 847-870. MR 26 \#622.

6. J. Dixmier, Les $C^{*}$-algèbres et leurs représentations, 2nd ed., Cahiers Scientifiques, fasc. 29, Gauthier-Villars, Paris, 1969. MR 39 \#7442.

7. N. Dunford and J. T. Schwartz, Linear operators. I: General theory, Pure and Appl. Math., vol. 7, Interscience, New York, 1958. MR 22 \#8302.

8. J. S. Pym, The convolution of functionals on spaces of bounded functions, Proc. London. Math. Soc. (3) 15 (1965), 84-104. MR 30 \#3367.

9. C. E. Rickart, General theory of Banach algebras, University Series in Higher Math., Van Nostrand, Princeton, N. J., 1960. MR 22 \#5903.

10. P.-K. Wong, On the Arens product and certain Banach algebras, Trans. Amer. Math. Soc. 180 (1973), 437-448. MR 47 \#7431.

DEPARTMENT OF MATHEMATICS, UNIVERSITY OF ABERDEEN, ABERDEENAB9 2UB, SCOTLAND 